Article

\title{
Functional T cell Reactivity to Melanocyte Antigens is Lost During Development of Malignant Melanoma, but is Restored with Successful Immunotherapy
}

\author{
Anna Przybyla1,2, Alexander A. Lehmann', Ting Zhang1, Jacek Mackiewicz ${ }^{3,4}$, Łukasz Galus ${ }^{3,4,5}$ \\ Greg A. Kirchenbaum 1, Andrzej Mackiewicz',4\#, Paul V. Lehmann" ${ }^{\# *}$ \\ 11 Research and Development Department, Cellular Technology Limited (CTL), Shaker Heights, OH, 44122, \\ USA \\ 2 Department of Cancer Immunology, Chair of Medical Biotechnology, Poznan University of Medical \\ Sciences, Poznan, Poland \\ 3 Department of Medical and Experimental Oncology, , University of Medical Sciences. Poznan, Poland \\ 4 Department of Diagnostics and Cancer Immunology, Greater Poland Cancer Center, Poznan, Poland \\ 5 Chemotherapy Department, Greater Poland Cancer Center, Poznan, Poland. \\ \# Contributed equally \\ * Correspondence: paul.lehmann@immunospot.com; Tel.: +1-216-965-6311
}

\begin{abstract}
Healthy human subjects develop spontaneous CD8+ T cell responses to melanocyte antigens (MA) expressed by normal melanocytes, such as Tyrosinase, MAGE-A3, Melan/Mart-1, gp100, and NY-ESO-1. This natural autoimmunity directed against melanocytes might confer protection against the development of malignant melanoma (MM), where MA are present as overexpressed tumor-associated antigens. Consistent with this notion we report here that functional $\mathrm{T}$ cell reactivity to MA was found to be diminished in untreated MM patients: while $57.5 \%$ of healthy controls $(\mathrm{n}=40)$ exhibited high-frequency MA-specific $\mathrm{T}$ cell reactivity ex vivo, such was detected in only $12 \%$ of the untreated MM patients $(n=24)$. Three lines of evidence suggest that the MA-reactive $\mathrm{T}$ cells present in healthy subjects undergo exhaustion once MM establishes itself. First, only the MA-specific T cell reactivity was affected in the MM patients; that to third party recall antigens was not. Second, in these patients, the residual MA-specific $\mathrm{T}$ cells, unlike third party antigen reactive $\mathrm{T}$ cells, were functionally impaired, showing a diminished per cell IFN- $\gamma$ productivity. Third, successful immunotherapy with AGI-101H melanoma vaccine restored natural CD8+ T cell autoimmunity to MA in $85 \%$ of the vaccinated patients $(n=27)$. The role of natural T cell autoimmunity to tumor-associated MA is discussed based on discrete levels of $\mathrm{T}$ cell activation thresholds.
\end{abstract}

Keywords: melanoma, genetic whole cell therapeutic melanoma vaccine (AGI-101H), CD8+ T cells; melanoma antigens; ELISPOT

Abbreviations: AGI-101H: genetic therapeutic melanoma vaccine, CEFpp32: a pool of 32 immune dominant peptides of Cytomegalovirus-, EBV and Flu virus; ELISPOT: Enzyme-Linked Immunospot Assay; gp100: melanoma glycoprotein 100; GzB: granzyme B, cytolytic secretory product of CD8+ T cells; HD: healthy donor; IFN- $\gamma$ : interferon gamma; IL-2: interleukin 2; MA: melanoma antigen; MM: melanoma malignum, MP: patient with malignant melanoma; MAGE-A3: melanoma-specific antigen A3; Melan-A/Mart-1: melanoma differentiation antigen; NY-ESO-1: melanoma antigen, member of the CT family of the cancer antigens; PBMC: peripheral blood mononuclear cells; SFU: spot forming unit; TNF- $\alpha$ : tumor necrosis factor alpha (cell signaling protein); TSCM: stem-cell-like CD8+ memory T cell; Tyr: Tyrosinase, a melanoma antigen. 


\section{Introduction}

It is well established that melanoma-specific CD8+ T cells can be detected in melanoma patients (MP) after the tumor has metastasized [1]. Melanoma is thought to be among the most immunogenic tumors due to its exceptionally high UV-driven mutational burden, allowing for the creation of unique neoantigens recognizable to T cells [2]. While the initial $\mathrm{T}$ cell response is likely to target these mutated "foreign" tumor-specific antigens, the anti-tumor T cell response apparently spreads [3-7], to involve unmodified "self" antigens, so called melanoma-associated antigens (MA). The latter can be classified as melanocyte lineage-specific antigens (MART-1/Melan-A, Tyrosinase, gp100) and antigens derived from genes expressed in testis and a variety of cancers (including MAGE-family, NY-ESO-1) [8]. CD8+ T-cell immunity directed against such MA is arguably the best-studied form of tumor-specific T-cell immunity in humans. Based on MHC multimer measurements, MA-specific CD8+ $\mathrm{T}$ cells can be detected in MP sometimes in substantial numbers [9], but their abundance is not accompanied by a clinically significant antitumor effect [10]. This apparently counter-intuitive finding can be reconciled with the notion that MA-specific CD8+ T cells in MP continuously progress from an early effector "transitional" into a dysfunctional T cell state with eventually dysfunctional T cells prevailing [11]. Thus, MA-specific CD8+ T cell are present, but become exhausted due to persistent antigen stimulation [12]. Such exhausted $T$ cells are characterized by the loss of classical CD8+ $\mathrm{T}$ cell effector functions, including their ability to kill, and to produce pro-inflammatory cytokines such as interferon-gamma (IFN- $\gamma$ ) [13, 14], resulting in part from their overexpression of inhibitory receptors such as programmed cell death (PD)-1 [15]. One of the major goals of cancer therapy is to restore tumor-specific CD8+ $\mathrm{T}$ cell effector functions by overcoming $\mathrm{T}$ cell exhaustion [16].

Most insights on MA-specific CD8+ T cells in MP have been gained using MHC multimers (tetramers/ pentamers/ dextramers). While these constructs are ideal for revealing the numbers of CD8+ T cells that are specific for a given MA peptide, along with the phenotype of the peptide-specific $\mathrm{T}$ cells, they are less suited to directly detect the actual functionality of such CD8+ $\mathrm{T}$ cells [17]. Exhausted CD8 T cells, however, comprise of heterogeneous cell populations with unique differentiation and functional states [13]. Because CD8+ T exhaustion is imprinted at an epigenetic level, it is inherited to daughter cells. Thus, temporary/partial therapeutic restauration of CD8+ T cells functions can occur in exhausted CD8+ T cells without fully reverting the phenotype; CD8 T cells may need to be epigenetically reprogrammed to achieve long-lasting reinvigoration [18]. Moreover, the multimer approach inherently is likely to cover only a fraction of the antigen-specific CD8+ T cell repertoire because CD8+ T cell responses can be restricted by all MHC class I molecules present in an individual, and they can recognize multiple epitopes on a given antigen [19]. Last but not least, multimers lack the sensitivity to reliably detect CD8+ $\mathrm{T}$ cell populations that occur in very low frequency, such as MA-specific CD8+ T cells in healthy individuals [20]. The functional MAspecific functional CD8+ T cell repertoire in healthy subjects, therefore, remained largely unexplored.

Relying on an ELISPOT approach that detects functional CD8+ T cells even as rare as 1:250,000 peripheral blood mononuclear cells (PBMC), and using mega peptide pools that cover all epitopes on MA, we have shown that in healthy human donors (HD) primed MA-specific CD8+ T cells can be detected [21]. Therefore, apparently not only melanoma cells are immunogenic after they have metastasized, but already regular melanocytes can trigger tumor associated antigen-specific $\mathrm{T}$ cell responses, and doing so without evidently harming regular melanocytes. In analogy to natural autoantibodies [22], that also should be forbidden based on the self/non-self model, we called these $\mathrm{CD} 8+\mathrm{T}$ cells natural autoreactive $\mathrm{T}$ cells. These MA-specific $\mathrm{T}$ cells recognize Tyrosinase, MAGEA3, Melan/Mart-1, gp100, and NY-ESO-1 [21]. Reminiscent of anti-viral T cell responses [19], the T cell response against these MA is aleatory [23], i.e., it is directed against different MA in different individuals [21]. These MA-specific natural CD8+ $\mathrm{T}$ cells are in a resting memory cell stage at their isolation form the body, but within 3 days of antigen re-stimulation they become cytolytic effector cells as resting memory cells generally do. 
It can be assumed that such naturally autoreactive CD8+ T cells get primed during sun burns we suffer when melanocyte activation occurs in an inflamed skin, and thus both requirements for priming of a $\mathrm{T}$ cell response are warranted: the temporary overexpression of the melanocyte antigens providing increased levels of TCR ligand sufficient to exceed the activation threshold of low affinity CD8 cells [24], and the "danger" environment required for the induction of immunity vs. of tolerance [25]. While such naturally autoreactive melanocyte-specific $\mathrm{T}$ cells might actively enhance the inflammatory response associated with the sun burn, and as such might in the long term promote melanoma development, in healthy hosts, they apparently become negligent of the autoantigen as soon as its expression level returns to normal as these cells are resting CD8 + T memory cells at their isolation [21]. But do such naturally autoreactive CD8 $+\mathrm{T}$ cells represent a novel line of immunity directed against melanoma?

In this communication we have addressed the question what happens to such fully functional naturally autoreactive MA-specific CD8 + T cells when melanoma develops. Overexpressed MA are now present in the host and thus should re-engage these $\mathrm{T}$ cells. Does the persistence of overexpressed melanocyte antigen lead to their recruitment in the anti-tumor effector cell pool, or, to the contrary, do such CD8 + T cells undergo exhaustion?

\section{Results}

\subsection{Natural T Cell Reactivity to Melanocyte Antigens in Healthy Donors}

As we reported before, healthy human donors (HD) frequently exhibit melanocyte antigen- (MA-) specific functional CD8+ T cells [21]. An example of such a recall response is provided in Figure 1A, showing a HD who gave a dominant recall response to MAGE-A3, and responded in a subdominant fashion to Tyrosinase, also a MA. In this HD the frequency of T cells responding to other MAs, namely

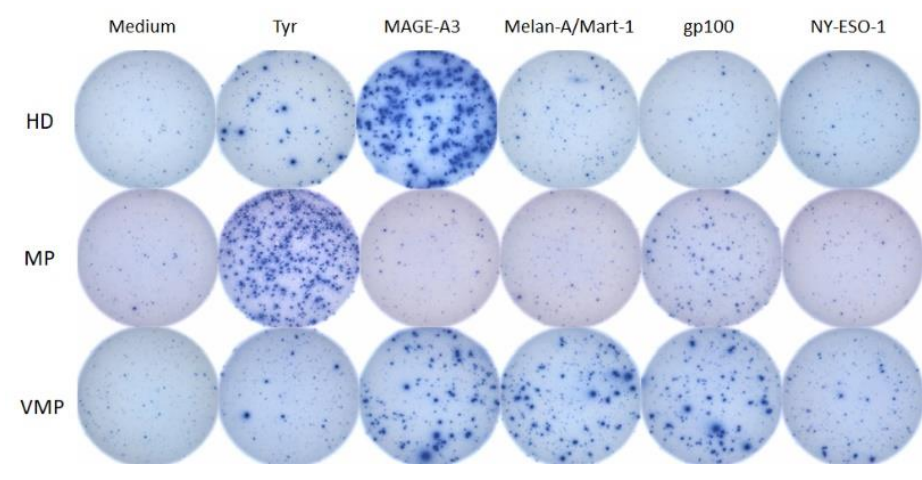

Figure 1. IFN- $\gamma$ ELISPOT recall responses to melanocyte antigens. The melanocyte antigens specified across the top were tested on PBMC of heathy donors (HD), untreated melanoma patients (MP) and MP vaccinated with AGI-101H (VMP). Well images are shown for a subject representative of the cohort.

namely Melan-A/Mart-1, gp100 and NY-ESO-1 was below the detection limit of the assay as performed, that is, indistinguishable from the medium control. Occasional subjects of the HD cohort $(n=40)$ responded to the latter MA as well (Figure 2A). As shown in Figure 3A, recall responses to at least one MA were detected in 23 of $40(57,5 \%) \mathrm{HD}$. The prevalent response type in HDs targeted a single MA (seen in 15 of $40 \mathrm{HD}, 37.5 \%$ ) whereas all five MA tested positive in three HDs in our cohort $(7.5 \%)$. Two and three MAs were recognized by 2 HDs each (5\%) and one of the $40 \mathrm{HD}$ responded to four MA. This MA-reactivity profile in the HD cohort serves as the reference against which we compared MA-reactivity in unvaccinated - and vaccinated melanoma patients. 
A.

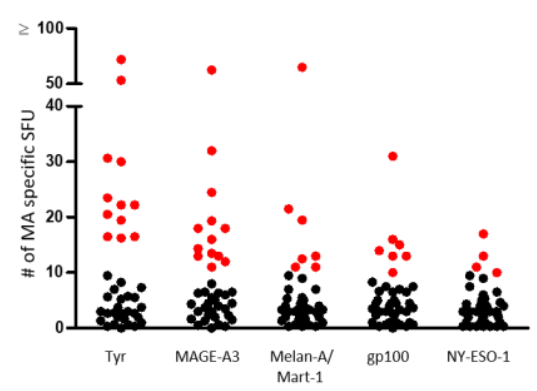

B.

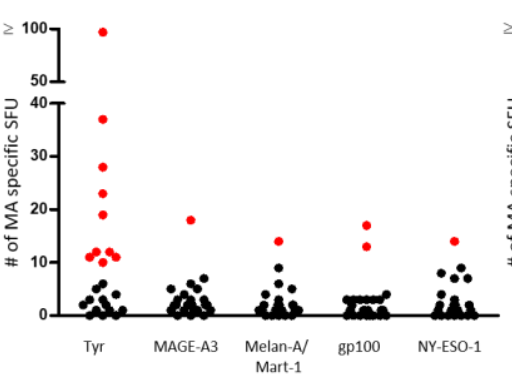

C.

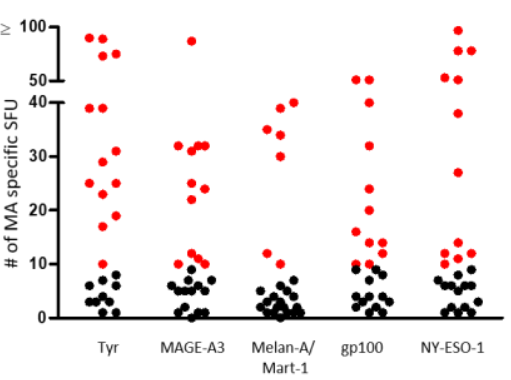

Figure 2. Number of melanocyte antigen-specific CD8+ T cells in PBMC of healthy donors $(H D, n=40)$, patients with untreated malignant melanoma $(M P, n=24)$ and MP vaccinated with AGI-101H (VMP, n=27). For each subject, the IFN$\gamma$ ELISPOT recall response was tested after exposing the PBMC to the melanocyte antigens specified on the $Y$ axis. Each data point represents the mean SFU count established in 250,000 PBMC/well, in triplicate wells. Positive T cell responses, as defined in Materials and Methods, are highlighted in red.

\subsection{Natural Melanocyte Antigen-Specific T Cell Immunity is Deficient in Patients with Untreated Malignant Melanoma}

We tested 24 melanoma patients (MP, all Stage IV, and pharmacologically untreated) for their recall responses to MA. In this cohort the MA-triggered recall responses were largely reduced compared to HC. Only two of the $24(8 \%)$ MP responded to MA, both to a single MA (Figure 3B). The MAinduced SFU counts were lower in MP than in HC (Fig. 2B vs. Fig. 2A). Additionally, the MAtriggered SFU size was decreased in the MP vs. the SFU sizes seen in HC (see in Figure 1B, MP's response to gp100 vs. the HC's response to MAGE-A3). Reduced SFU sizes reveal impaired per T cell IFN- $\gamma$ secretion [30] being a characteristic of T cells that have developed partial anergy [14]. These
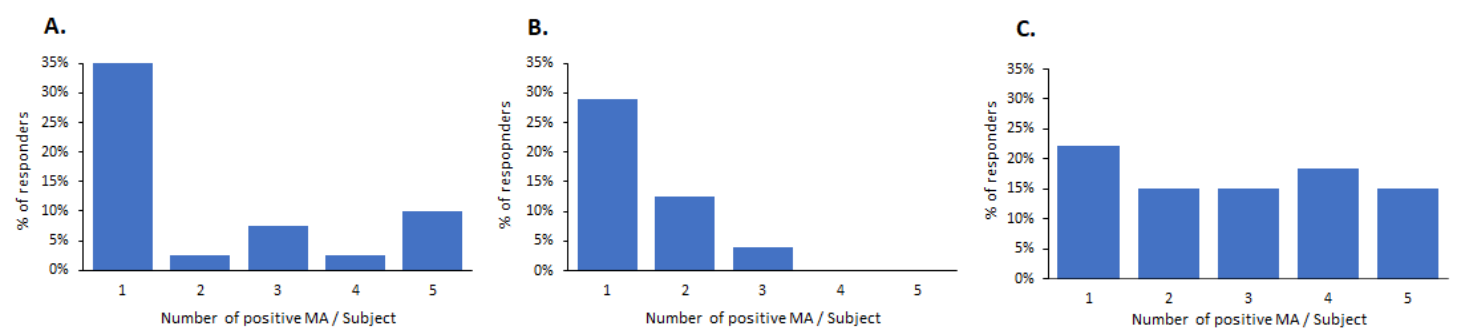

Figure 3. Number of melanocyte antigens (MA) eliciting positive T cell responses in (A) healthy donors (HD, $n=40),(B)$ patients with untreated malignant melanoma $(M P, n=24)$, and MP vaccinated with AGI-101H (VMP, n=27). PBMC of each subject were tested in an IFN- $\gamma$ ELISPOT assay for T cell reactivity to five MA: Tyrosinase, MAGE-A3, MelanA/Mart-1, gp100, and NY-ESO-1. The number of MA that elicited a positive response per donor (X axis) is shown vs. the percentage of subjects in each cohort responding to that number of $M A$, specified on the $Y$ axis.

changes in MP's $\mathrm{T}$ cell responses were MA-specific as the MP's $\mathrm{T}$ cell recall response to third party recall antigens, CEF peptides, was not impaired compared to the HC cohort (Figure 4). The CEF peptide pool consists of immune dominant epitopes of Cytomegalo-, Epstein Barr- and Flu Virus [27]. 


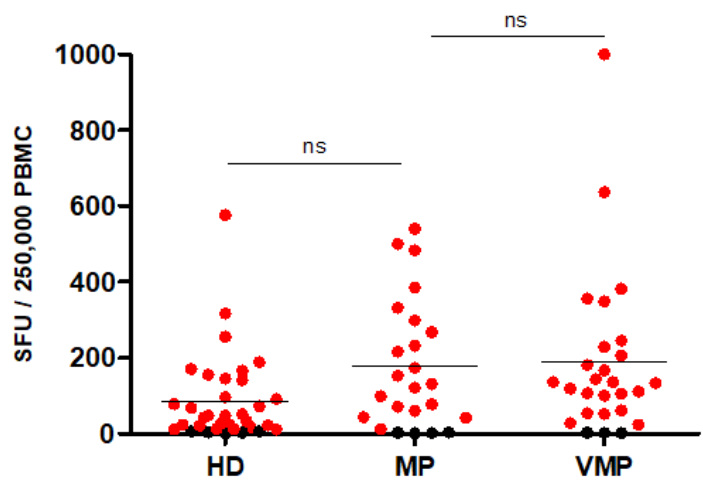

Figure 4. Number of CEF peptide-reactive CD8+ T cells in PBMC of healthy donors (HD), patients with untreated malignant melanoma (MP), and MP vaccinated with AGI-101H (VMP). For each subject, the IFN- $\gamma$ ELISPOT recall response was tested after exposing the PBMC to the CEF peptide pool. Each data point represents the mean SFU count established in triplicate wells; positive CEF-peptide-induced responses are highlighted in red. The non-parametric MannWhitney $U$ test did not reveal statistically significant differences between the cohorts.

\subsection{Successful vaccination restores natural $T$ cell autoimmunity in melanoma patients}

AGI-101H is a melanoma vaccine that relies on super-IL-6 transfected allogeneic melanoma cells [31]. In phase 2 clinical trials AGI-101H was shown to have a 50\% response rate on Stage III and Stage IV melanoma patients with those responding surviving up to 15 years [32-34].We tested 27 such successfully AGI-101H vaccinated melanoma patients (VMP) for their $\mathrm{T}$ cell responses to MA. Twenty three of $27(85 \%)$ subjects in the VMP cohort responded to MA with SFU response magnitudes of MA-specific T cells comparable to that in HD (Figure $2 \mathrm{C}$ vs. Figure 2A). These VMP subjects commonly targeted several MA (Fig. 3C): four of the 27 VMP (14.8\%) responded to all five MA tested, five displayed T cell responses to four MA $(18.5 \%)$, three $(11 \%)$ responded to three MA, dual MA recognition was seen in 5 subjects (18.5\%) while single MA recognition occurred in $22 \%$ of the VMP (6 of 27). Original data for a representative VMP are shown in Figure 1C. This subject displayed strong recall responses to MAGE-A3, Melan/Mart-1 and gp100 and weaker responses to Ny-Eso-1 and Tyrosinase. The described changes in VMP's T cell responses were MA-specific as this cohort's $\mathrm{T}$ cell recall response to third party recall antigens was unimpaired compared to HC and MP (Figure 4). Vaccination with IL-6 transfected allogeneic melanoma cells (the AGI-101H vaccine) therefore not only successfully restored $\mathrm{T}$ cell reactivity to MA in MP, but raised it beyond the level present in HC. Subsection

\section{Discussion}

The first set of data presented here establish that in untreated MP natural T cell reactivity to MA is severely reduced. A similar observation was reported for breast cancer: HD were shown to respond the breast cancer associated antigen HER-2, but breast cancer patients selectively lacked this reactivity [35]. At this point, there are two possible interpretations for the lack of natural $\mathrm{T}$ cell autoreactivity in cancer patients. One is, that pre-existing autoimmunity protects against cancer development, and subsequently in cancer patients the non-responder phenotype is prevalent. An alternative, and equally plausible interpretation for the lack of natural $\mathrm{T}$ cell autoreactivity in melanoma (and breast cancer) patients is the selective inactivation of these $\mathrm{T}$ cells: the persisting tumor will continue to stimulate and eventually exhaust them.

Beyond establishing the fact of absent natural $\mathrm{T}$ cell autoreactivity in melanoma, our data do not permit to distinguish between the above two possibilities. The first interpretation is simple, and may not merit in depth discussion. Accordingly, individuals lacking natural anti-MA T cell immunity have developed melanoma and their immunization with an allogenic melanoma vaccine induces protective anti-MA immunity. As this vaccine is allogenic, but we used a self-restricted detection 
system, cross-presentation must have triggered the anti-MA $\mathrm{T}$ cell response detected in the VMP subjects. However attractive this first hypothesis is due to its simplicity, the data can also be interpreted in the context of multiple discrete $\mathrm{T}$ cell activation threshold levels operating in the three cohorts. Because of its complexity the latter hypothesis may merit more deliberation.

$\mathrm{T}$ cell activation occurs when a signaling threshold is reached that is primarily dictated by the extent of the T cell Receptor's (TCR) ligation [24]. In reaching this threshold, the TCR's affinity for the TCR-ligand (TCR-L, consisting of the nominal antigen peptide " $\mathrm{X}$ " presented on an MHC molecule) is reciprocally related to the TCR-L density on the antigen-presenting cell (APC): $T$ cell clones with high affinity TCR for TCR-L X will become activated even at low densities of TCR-LX on the APC, while T cells with a TCR that has low affinity for TCR-LX need TR-LX to be present in high density [36]. In the context of autoantigen (and hence tumor associated antigen) recognition by $\mathrm{T}$ cells it is primarily the level of autoantigen expression that defines the density of TCR-LX on the APC, thus defining whether a T cell's activation threshold is reached.

In the case of MA-specific T cells, the first discrete TCR-L threshold level ("Level 1") is set by the constitutive expression of MA in the thymus and on resting melanocytes. During negative selection, the MA-specific T cell repertoire is trimmed off clones whose TCR is of high enough affinity to become activated by the Level-1 antigen presentation threshold, while MA-specific clones of lower affinity surviving as naïve T cells that are "ignorant" of the autoantigen [37]. Precursor cells for MAspecific effector T cells should fall in this > Level 1 TCR affinity category.

During sun burns the skin gets inflamed and melanocytes become mobilized now expressing MA at a higher level than resting melanocytes do. This results in an increased TCR-L level on activated melanocytes (Level-2): T cell clones whose TCR affinity for MA is high enough to reach the activation threshold by Level-2 TCR-L become primed to effector/memory T cells. We assume that such $\mathrm{T}$ cells represent the naturally autoreactive CD8+ T cells we have observed in HD. These T cells can target inflamed melanocytes, but they cannot attack resting melanocytes as TCR-L density on the latter is subthreshold for them, and as such, they pose no autoimmune threat. Once the skin inflammation subsides, TCR-L expression again settles in at Level 1, and the naturally autoreactive T cells, now memory cells, become ignorant of the autoantigen. Subsequently the naturally autoreactive CD8 T cells in HD do not undergo exhaustion. Matching this hypothesis, at isolation they were found to be fully functional, resting memory cells, being IFN- $\gamma$ positive, granzyme B negative [21].

MA are overexpressed in melanoma cells compared to melanocytes [38], resulting in Level 3 TCR-L density. Exceeding their > Level 1 activation threshold, naturally autoreactive CD8 cells will become stimulated by melanoma cells. Such T cells might kill off melanoma cells and protect from melanoma development altogether. If the tumor persists, after initially combatting the tumor, these naturally autoreactive $\mathrm{T}$ cells are likely to be driven by the persisting antigen stimulation into a state of partially reversible unresponsiveness (anergy) and eventually into exhaustion and senescence [14]. Subsequently, the naturally autoreactive $\mathrm{T}$ cells become undetectable in patients with melanoma when their pro-inflammatory effector function is measured by IFN- $\gamma$ ELISPOT, while according to the literature they should be still detectable as multimer-positive, but exhausted T cells.

$\mathrm{T}$ cells that have TCR affinities exceeding the Level 2 TCR-L threshold will recognize MA on melanoma cells only, to them Level-2 presentation on inflamed or regular melanocytes is subthreshold. Although such Level 3-reactive T cells recognize autoantigens that in terms of amino acid sequence are shared between tumor- and normal cells, they are exquisitely tumor-specific due to TCR affinity-based expression level discrimination: only the > Level 2 TCR-L density on the tumor cells will meet their activation threshold. 
We found that, unlike unvaccinated MP, MP patients who have undergone successful AGI-101H vaccination showed strong recall responses to MA. The allogeneic IL-6 transfected melanoma cells that constitute the vaccine apparently have succeeded to trigger MA-specific $\mathrm{T}$ cell immunity in these subjects. At this point one can-not tell whether exhausted Level-2 CD8+ T cells were re-activated by the vaccine, or whether Level-3-reactive CD8 cells were primed that had been naïve in MP prior to vaccination, or both. Possible mechanisms for the reinvigoration of exhausted CD8+ T cells include PD-1 level reduction, increased CD28 costimulation, cytokine effects, and reactivation of the stem cell-like CD8 subset, all of which can jointly or independently reinstate effector functions of exhausted T cells (Reviewed in [18]). Of these, the likelihood of the reactivation of the stem cell-like CD8 subset strikes us likely. Stem cell-like CD8+ T cells are abundant in the MA-reactive T cell repertoire of HD [21]. Stem cell-like CD8 T cells are present predominantly in the T cell zones of lymphoid tissues, and are likely to continuously interact with dendritic cells in the T cell zones [18]. The AGI-101H vaccine is administered subcutaneously, delivering MA to the regional lymph nodes. The IL-6 expressed by the injected allogeneic vaccine cells, plus the local inflammatory environment triggered by allo-reactive host $\mathrm{T}$ cells might contribute to the reactivation of the stem cell-like CD8+ $\mathrm{T}$ cells. IL-21 has been vital for sustaining CD8 T cell responses avoiding their exhaustion [39-41]. IL-2 has a demonstrated efficacy on melanoma [42]. While the exact mechanism of AGI-101H vaccine's action will need to be established, the data presented here demonstrate that anti-MA immunity that went functionally dormant in unvaccinated MP has become re-engaged in the VMP, and the clinical efficacy of the vaccine suggest that these MA specific T cells might also contribute to the immune surveillance of this cancer.

\section{Materials and Methods}

\subsection{Human Subjects}

4.1.1. Healthy Controls. Peripheral blood mononuclear cells (PBMC) from healthy human donors (HD) were selected from the ePBMC library (CTL, Shaker Heights, OH, USA). The PBMC had been collected by HemaCare Blood Donor Center (Van Nuys, CA) under HemaCare's IRB and sold to CTL identifying donors by code only while concealing the subjects' identities. The characteristics of the randomly selected forty HD are specified in Supplemental Table 1.

4.1.2. Untreated Melanoma Patients. PBMC were isolated from 24 advanced melanoma patients (MP), all in stage IV of the disease with resected or non-resected metastatic melanoma. These patients have not been treated with any immunotherapy before the blood collection. For patients with resected melanoma, sampling was performed following surgical resection of metastases while for patients with non-resectable disease, sampling was carried out prior the enrollment to another treatment. Four patients underwent the surgical removal of the metastasis followed by radiotherapy completed before blood drawing. All patients gave written informed consent for blood donation and PBMC collection. The clinical characteristics of these MP are summarized in Supplemental Table 2.

4.1.3. Melanoma Patients treated with AGI-101H vaccine. The AGI-101H vaccine consists of two melanoma cell lines (Mich-1 and Mich-2) that were retrovirally transduced with a fusion protein of interleukin 6 (IL-6) and its agonistic soluble IL-6 $\alpha$ receptor [31]. Patients with advanced melanoma, with resected or non-resected metastases, were injected subcutaneously with AGI-101H ( $5 \times 107$ cells/dose $) 8$ times in 2 week intervals (induction phase), then every month (maintenance phase) indefinitely or until death (Clinical Trial ETAM2-5). In case of recurrence, the induction phase was repeated with or without surgery and followed by maintenance. A significant number of AGI-101H-treated patients (vaccinated melanoma patients, VMP) are still alive - out of the 138 melanoma patients enrolled in ETAM2-5 Trial, $96(69.6 \%)$ survived a mean treatment duration of 196 months (ranging from 144 to 245 months). Twenty-three survivors from the ETAM2-5 Trial were randomly selected to participate in this study along with four melanoma patients enrolled to the AGI-101H vaccination schedule in 2015. At the time of blood drawing, all patients treated with vaccine AGI-101H were free of clinically manifested disease. 
The ETAM trial was approved by the RBC and the Central Evidence of Clinical Trials (EnduraCT Number 2008-003373-40). All patients had to provide the signed informed consent for the participation in the study before any study-related procedures. The clinical characteristics of the twenty-seven AGI101H-treated melanoma patients are summarized in Supplemental Table 3.

\subsection{Peripheral blood mononuclear cell preparation}

Blood was obtained by venipuncture under sterile conditions in heparinized tubes (BD Biosciences, Franklin Lakes, N. J., U.S.A) and PBMC were isolated within 3 h by standard gradient centrifugation over Histopaque 1077 (Sigma-Aldrich, St. Louis, MO, USA). The PBMC were cryopreserved within $1 \mathrm{~h}$ using CTL-Cryo ${ }^{\mathrm{TM}}$ ABC Media Kit (CTL, Shaker Hts, OH., USA) and stored in the vapor phase of liquid nitrogen until testing. Thawing, washing, and counting of PBMC were performed according to CTL protocols [26]. The PBMC were suspended at a final concentration of 2.5 million PBMC/mL in CTL-Test Medium (CTLT-005) of which $100 \mu \mathrm{L}$ (250,000 PBMC) were plated per well into the ELISPOT assays within $2 \mathrm{~h}$ after thawing.

\subsection{Antigens}

Five previously described [21] melanoma-associated antigens (MA) were tested in this study, each represented by a peptide pool consisting of 15-mer peptides that cover the entire amino acid sequence of the respective human protein in steps of 11 amino acids. All five MA peptide pools were purchased from JPT (Berlin, Germany): Tyrosinase (PM-Tyr), MAGE-A3 (PM-MAGEA3); Melan-A/Mart-1 (PMMelan-A); Melanocyte protein Pmel 17gp100 (PM-GP100), and NY-ESO-1 (PM-NYE). All five MA peptide pools were tested at a final concentration of $1 \mu \mathrm{g} / \mathrm{mL}$. As a positive control for antigen-specific $\mathrm{T}$ cell activation we used CEF (from CTL, Cat \#: CTL-CEF-002) that consists of a pool of 32 immune dominant nonamer peptides derived from CMV, EBV and Flu virus [27]. The CEF pool was tested at a final concentration of $0.25 \mu \mathrm{g} / \mathrm{mL}$. All peptides were dissolved in CTL-Test Medium.

\subsection{IFN- $\gamma$ ImmunoSpot® Assay}

Human IFN- $\gamma$ single-color enzyme-linked immunospot (ELISPOT/ImmunoSpot) assays were from CTL, and were performed following the manufacturer's protocol. In brief, the PVDF membrane was pre-coated with IFN- $\gamma$ capture antibody overnight. After three washes, the antigens, dissolved in CTL-Test Medium, were plated in $100 \mu \mathrm{L}$ per well. The plates with the antigen were stored at $37{ }^{\circ} \mathrm{C}$ in a CO2 incubator until the cells were ready for plating. Within an hour, the freshly thawed PBMC were added at 250,000 cells/well in $100 \mu \mathrm{L}$ using wide-bore pipette tips to avoid shear forces damaging the cells. The plates then were gently tapped on each side to ensure an even sedimentation of the PBMC in the wells. The cells were incubated with the antigens for $72 \mathrm{~h}$ in a humidified incubator at $37{ }^{\circ} \mathrm{C}$ and $9 \% \mathrm{CO} 2$. After this activation culture during which IFN- $\gamma$ is captured around each antigen-stimulated $\mathrm{T}$ cell, the cells were removed, IFN- $\gamma$ detection antibody was added, and the plate-bound cytokine spots were visualized by enzyme-catalyzed substrate precipitation. The plates were air-dried prior to analysis. The results were analyzed using an ImmunoSpot ${ }^{\circledR}$ S6 Ultimate Reader from CTL. Spot Forming Units (SFU), each representing the secretory footprint of a single IFN- $\gamma$ secreting $\mathrm{T}$ cell, were automatically counted by the ImmunoSpot ${ }^{\circledR}$ Software (CTL) that relies on statistics-based automatic setting of upper and lower size gates for SFU counting [28].

\subsection{Statistical analysis}

ELISPOT counts follow normal distribution among replicate wells which permits reliance on parametric statistics for identifying positive responses [29]. Accordingly, the Student's t-test was done comparing for each test subject, and test, SFU counts in three antigen-containing replicate wells vs. the spot counts in three medium control wells. A p value $<0.01$ was considered as the cut-off for positivity. For comparing response magnitudes among cohorts, the non-parametric Mann-Whitney U test was 
performed considering $\mathrm{p}$ values $<0.01$ statistically significant. Interventionary studies involving animals or humans, and other studies require ethical approval must list the authority that provided approval and the corresponding ethical approval code.

Supplementary Materials: The following are available online at www.mdpi.com/xxx/s1, Table S1: Characteristics of healthy donors (HD) tested in this study; Table S2: Characteristics of untreated melanoma patients (MP) tested in this study; Table S3: Characteristics of melanoma patients treated with the AGI-101H vaccine (VMP) tested in this study.

Author Contributions: All authors have read and agreed to the published version of the manuscript. PVL designed and supervised the ex vivo T cell immune monitoring. A.M. the clinical study with J.M and E.G contributing to the clinical trial. The experiments were conducted by A.P with A.A.L, T.Z and G.K contributing. The manuscript was written by P.V.L and A.P. This publication serves as part of A.L.'s doctoral thesis to be submitted to the Universidad Complutense de Madrid, Madrid, Spain

Funding: The ex vivo $\mathrm{T}$ cell immune monitoring part of this study was conducted in the Research and Development Department of Cellular Technology Limited (CTL) and was funded by the research budget of CTL. The clinical part of the study was performed at Poznan University of Medical Sciences, and was funded by Innomed Project: Personalization of melanoma therapeutic vaccination (Innomed/I/6/NCBR/2014).

Acknowledgments: The authors would like to thank Katarzyna Tomela and Katarzyna Gryska for helping with PBMC's collection, Marzena Libera for clinical data collection and Diana Roen for expert editorial assistance.

Conflicts of Interest: P.VL., T.Z., A.A.L., and G.A.K. are employees of Cellular Technology Limited (CTL), a company that specializes in ELISPOT testing, producing high-throughput-suitable readers, test kits, and GLPcompliant contract research. A.M., A.P., J.M. and L.G., are employees of Poznan University of Medical Sciences. A.M. and L.G. are employees of Greater Poland Cancer Centre, Poznan, Poland as well. The authors declare no financial, commercial or other relationships that might be perceived by the academic community as representing a potential conflict of interest.

\section{References}

1. Passarelli A. MF, Stucci LS, Tucci M, Silvestris F. Immune system and melanoma biology: a balance between immunosurveillance and immuneescape. Oncotarget. 2017;31:106132-42; 10.18632/oncotarget.22190.

2. Ko JS. The immunology of melanoma. Clinics in Laboratory Medicine. 2017;37:449-71; 10.1016/j.cll.2017.06.001.

3. Lehmann PV, Forsthuber T, Miller A, Sercarz EE. Spreading of T-cell autoimmunity to cryptic determinants of an autoantigen. Nature. 1992 Jul 9;358(6382):155-7. 10.1038/358155a0.

4. Schlingmann TR, Rininsland FH, Bartholomae WC, Kuekrek H, Lehmann PV, Tary-Lehmann M. Vaccination with tumor cells pulsed with foreign peptide induces immunity to the tumor itself. Clinical Immunology. 2009;133:45-51; 10.1016/j.clim.2009.06.004.

5. Corbiere V, Chapiro J, Stroobant V, Ma W, Lurquin C, Lethe B, et al. Antigen spreading contributes to MAGE vaccination-induced regression of melanoma metastases. Cancer Research. 2011;71:1253-62;10.1158/00085472.CAN-10-2693.

6. Kreiter S, Vormehr M, van de Roemer N, Diken M, Lower M, Diekmann J, et al. Mutant MHC class II epitopes drive therapeutic immune responses to cancer. Nature. 2015;520:692-6;10.1038/nature14426.

7. Ott PA, Hu-Lieskovan S, Chmielowski B, Govindan R, Naing A, Bhardwaj N, et al. A Phase Ib trial of personalized neoantigen therapy plus anti-PD-1 in patients with advanced melanoma, non-small cell Lung cancer, or bladder cancer. Cell. 2020;183:347-62; e24;10.1016/j.cell.2020.08.053.

8. Boon T CJ, Van den Eynde B, van der Bruggen P, Van Pel A. Tumor antigens recognized by T lymphocytes. Annu. Rev. Immunol. 1994;12:337-65; 10.1146/annurev.iy.12.040194.002005 
9. Lee PP YC, Savage PA, Fong L, Brockstedt D, Weber JS, Johnson D, Swetter S, Thompson J, Greenberg PD, Roederer M, Davis MM. Characterization of circulating $\mathrm{T}$ cells specific for tumor-associated antigens in melanoma patients. Nat. Med 1999;5:677-85; 10.1038/9525.

10. van Oijen M BA, Elias S, Sein J, Weder P, de Gast G, Mallo H, Gallee M, Van Tinterten H, Schumacher T, Haanen J. On the role of melanoma-specific CD8+ T cell immunity in disease progression of advanced-stage melanoma patients. Clinical Cancer Research. 2004; 2004; 10:4754-60; 10.1158/1078-0432.CCR-04-0260.

11. Li H, van der Leun AM, Yofe I, Lubling Y, Gelbard-Solodkin D, van Akkooi ACJ, et al. Dysfunctional CD8 T cells form a proliferative, dynamically regulated compartment within human melanoma. Cell. 2019;176:775-89 e18;10.1016/j.cell.2018.11.043.

12. Kurachi M. CD8+ T cell exhaustion. Seminars in Immunopathology. 2019;41:327-37;10.1007/s00281-019-00744.

13. Wherry EJ, Kurachi M. Molecular and cellular insights into T cell exhaustion. Nature Reviews Immunology. 2015;15:486-99;10.1038/nri3862.

14. Crespo J, Sun H, Welling TH, Tian Z, Zou W. T cell anergy, exhaustion, senescence, and stemness in the tumor microenvironment. Current Opinion in Immunology. 2013;25:214-21; 10.1016/j.coi.2012.12.003.

15. Sharpe AH, Pauken KE. The diverse functions of the PD1 inhibitory pathway. Nature Reviews Immunology. 2018;18:153-67;10.1038/nri.2017.108.

16. Pauken KE, Wherry EJ. Overcoming T cell exhaustion in infection and cancer. Trends in Immunology. 2015; 36:265-76;10.1016/j.it.2015.02.008.

17. Rubio-Godoy V, Dutoit V, Rimoldi D, Lienard D, Lejeune F, Speiser D, et al. Discrepancy between ELISPOT IFN-gamma secretion and binding of A2/peptide multimers to TCR reveals interclonal dissociation of CTL effector function from TCR-peptide/MHC complexes half-life. Proc. Natl. Acad. Sci. U S A. 2001; 98:103027;10.1073/pnas.181348898.

18. Hashimoto M, Kamphorst AO, Im SJ, Kissick HT, Pillai RN, Ramalingam SS, Araki K, Ahmed R. CD8 T cell exhaustion in chronic infection and cancer: opportunities for interventions. Annu. Rev. Med. 2018; 69 301-18; 10.1146/annurev-med-012017043208.

19. Lehmann PV, Suwansaard M, Zhang T, Roen DR, Kirchenbaum GA, Karulin AY, et al. Comprehensive evaluation of the expressed CD8+ T cell epitope space using high-throughput epitope mapping. Frontiers in Immunology. 2019;10:655;10.3389/fimmu.2019.00655.

20. Palermo B CR, Montovani S, Lantelme E, Manganoni AM, Carella G, Da Prada GA, Robustelli della Cuna G, Romagne F, Gauthier L, Necker A, Giachino C. Diverse expansion potential and heterogeneous avidity in tumorassociated antigen-specific T lymphocytes from primary melanoma patients. Eur. J. Immunol. 2001;31:412-20.

21. Przybyla A, Zhang T, Li R, Roen DR, Mackiewicz A, Lehmann PV. Natural T cell autoreactivity to melanoma antigens: clonally expanded melanoma-antigen specific CD8+ memory T cells can be detected in healthy humans. Cancer Immunology, Immunotherapy 2019;68:709-20;10.1007/s00262-018-02292-7.

22. Lobo PI. Role of natural autoantibodies and natural IgM anti-leucocyte autoantibodies in health and disease. Frontiers in Immunology. 2016; 7:198;10.3389/fimmu.2016.00198.

23. Lehmann AA, Lehmann P.V. Aleatory epitope recognition prevails in human $\mathrm{T}$ cell responses? Critical Reviews in Immunology 2020; 40:225-35;10.1615/critrevimmunol.2020034838.

24. Viola A Lanzavecchia A. T cell activation determined by T cell receptor number and tunable thresholds. Science. 1996; 5:104-8; 10.1126/science.273.5271.104

25. Matzinger P. The danger model: a renewed sense of self. Science. 2002; 296:301-5; 10.1126/science.1071059. 
26. Ramachandran H, Laux J, Moldovan I, Caspell R, Lehmann PV, Subbramanian RA. Optimal thawing of cryopreserved peripheral blood mononuclear cells for use in high-throughput human immune monitoring studies. Cells. 2012; 1:313-24;10.3390/cells1030313.

27. Currier JR KE, Turk E, Earhart LB, Loomis-Price L, Janetzki S, Ferrari G, Birx DL, Cox JH. A panel of MHC class I restricted viral peptides for use as a quality control for vaccine trial ELISPOT assays. J Immunol. Methods. 2002; 260:157-72; 10.1016/s0022-1759(01)00535-x.

28. Zhang W, Lehmann PV. Objective, user-independent ELISPOT data analysis based on scientifically validated principles. Methods in Molecular Biology. 2012; 792:155-71;10.1007/978-1-61779-325-7_13.

29. Karulin AY, Caspell R, Dittrich M, Lehmann PV. Normal Distribution of CD8+ T-Cell-Derived ELISPOT Counts within replicates justifies the reliance on parametric statistics for identifying positive responses. Cells. 2015; 4:96-111;10.3390/cells4010096.

30. Karulin AY, Lehmann PV. How ELISPOT morphology reflects on the productivity and kinetics of cells' secretory activity. Methods in Molecular Biology. 2012;792:125-43;10.1007/978-1-61779-325-7_11.

31. Mackiewicz A, Wysocki PJ, Suchorska W. Vaccine composition US patent US2011002898.

32. Mackiewicz A MJ, Wysocki PJ. Long-term survival of high-risk melanoma patients immunized with a HyperIL-6-modified allogeneic whole-cell vaccine after complete resection. Expert. Opin. Investig. Drugs 2012;21:77383.

33. Mackiewicz J, Karczewska-Dzionk A, Laciak M, Kapcinska M, Wiznerowicz M, Burzykowski T, et al. Whole cell therapeutic vaccine modified with hyper-IL6 for combinational treatment of non-resected advanced melanoma. Medicine. 2015;94:e853;10.1097/MD.0000000000000853.

34. Mackiewicz J, Burzykowski T, Izycki D, Mackiewicz A. Re-induction using whole cell melanoma vaccine genetically modified to melanoma stem cells-like beyond recurrence extends long term survival of high risk resected patients - updated results. Journal for Immunotherapy of Cancer. 2018; 6:134; 10.1186/s40425-018-04561 .

35. Datta J, Rosemblit C, Berk E, Showalter L, Namjoshi P, Mick R, et al. Progressive loss of anti-HER2 CD4+ Thelper type 1 response in breast tumorigenesis and the potential for immune restoration. Oncoimmunology. 2015;4:e1022301;10.1080/2162402X.2015.1022301.

36. Targoni OS, Lehmann PV. Endogenous myelin basic protein inactivates the high avidity T cell repertoire. J. Exp. Med. 1998;187:2055-63; 10.1084/jem.187.12.2055.

37. Ohashi PS DA. Making and breaking tolerance. Current Opinion in Immunology. 2002;14:744-59; 10.1016/S0952-7915(02)00406-5.

38. Schumacher TN SR. Neoantigens in cancer immunotherapy. Science. 2015;348:69-74; 10.1126/science.aaa4971 39. Frohlich A, Kisielow J, Schmitz I, Freigang S, Shamshiev AT, Weber J, et al. IL-21R on T cells is critical for sustained functionality and control of chronic viral infection. Science. 2009;324:1576-80; 10.1126/science.1172815.

40. Elsaesser H, Sauer K, Brooks DG. IL-21 is required to control chronic viral infection. Science. 2009;324:1569$72 ; 10.1126 /$ science. 1174182 .

41. Yi JS, Du M, Zajac AJ. A vital role for interleukin-21 in the control of a chronic viral infection. Science. 2009;324:1572-6; 10.1126/science.1175194.

42. Jiang T, Zhou C, Ren S. Role of IL-2 in cancer immunotherapy. Oncoimmunology. 2016;5:e1163462; 10.1080/2162402X.2016.1163462. 